\title{
Star-disk interaction in brown dwarfs: implications for substellar formation
}

\author{
Subhanjoy Mohanty \\ Harvard-Smithsonian CfA, 60 Garden Street, M.S.42, Cambridge, MA 02138, USA \\ email: smohanty@cfa.harvard.edu
}

\begin{abstract}
I review the current state of knowledge regarding disk accretion in young brown dwarfs (BDs), and the interaction of the disk with the central object. In particular, I discuss (1) observations of accretion/outflow phenomena in BDs; (2) techniques for measuring accretion rates $\left(\dot{M}_{a c c}\right) ;(3)$ the dependence of $\dot{M}_{a c c}$ on the central mass from stars to brown dwarfs; (4) the temporal evolution of $\dot{M}_{a c c}$; and (5) observations of variability in the accretion line profiles. I then examine the implications of these issues for the formation mechanism of BDs, and discuss new observations that can further constrain substellar origins.
\end{abstract}

Keywords. Circumstellar matter, planetary systems, stars: formation, stars: low-mass, brown dwarfs, stars: pre-main-sequence.

\section{Introduction}

Brown dwarfs (BDs) are by definition substellar objects: with a mass $\lesssim 80 \mathrm{M}_{\text {Jup }}(0.08$ $\left.\mathrm{M}_{\odot}\right)$, they are incapable of sustaining stable hydrogen fusion, and thus, after an initial period of Deuterium fusion, simply continue to cool down and grow fainter with time. Over the last decade, astronomers have discovered hundreds of these diminutive bodies, both in the field as well as in star-forming regions and young clusters, with masses ranging down to nearly (or perhaps even below) the planetary mass boundary $\left(\sim 12 \mathrm{M}_{\text {Jup }}\right.$ ). While some BDs are companions to stars, the vast majority are isolated bodies.

The formation mechanism of these ultra-low mass objects presents a challenge to theorists. The central dilemma is easily framed: since the average thermal Jeans mass in molecular clouds is usually of order a solar mass, how does one form free-floating objects ten to a hundred times less massive? Two main competing hypotheses have emerged. The 'ejection' scenario posits that BDs are in fact 'stellar embryos', born within molecular cloud cores but subsequently flung out from the cores by dynamical interactions with their neighbours before accumulating enough gas to become full-fledged stars (Reipurth \& Clarke 2001; Bate et al. 2003). A key prediction here is that disks around young BDs should be severely truncated by these interactions, and hence far smaller than those girdling newborn stars. As a corollary, the classical T Tauri (CTT) phase of disk accretion that is ubiquitous in young stars may also be shortlived and rare in BDs.

In the alternative 'turbulent fragmentation' picture (Padoan \& Nordlund 2004), turbulent shocks in molecular clouds create gravitationally bound cores with a range of masses: from large ones that form stars, to ultra-low mass ones - far below the cloud's mean thermal Jeans mass, but locally unstable - that collapse directly into BDs. In this view, there is no fundamental distinction between the formation mechanism of stars and BDs: both form quietly within their own cores. Extended disks, and an associated CTT phase, should then be as common in BDs as in stars.

These two formation mechanisms have key implications for low-mass star formation in general as well. In the first case, gravitational interactions also set the final stellar 
masses, by limiting the amount of material that can accrete onto nascent protostars: through competitive accretion, repeated truncation of the primordial disks, and outright ejection from the core. In the second case, the mass spectrum of star-forming cores is ultimately regulated by cloud turbulence. Thus, discriminating between these substellar formation scenarios is also vital for understanding the physics governing the stellar initial mass function (IMF).

The search for disks, and a CTT-like disk-accretion phase, around young BDs has consequently been a major area of research in the last few years. Disk studies have focussed on excess dust emission at near-infrared to sub- $\mathrm{mm} / \mathrm{mm}$ wavelengths, while the identification of disk-accretion relies primarily on optical and near-infrared spectroscopy. Surveys have now firmly established that disks are very common in young BDs, just as in stars. In this paper, I review the accretion results.

It is worth pointing out at this juncture that simply the presence of disks and accretion, while providing constraints on BD formation, is not sufficient to unambiguously distinguish between the two formation scenarios discussed above. I return to this subject in $\S 5$ and 6 , where I review the constraints implied by current data, and if and how future observations combined with current ones may finally settle the issue of $\mathrm{BD}$ formation.

\section{Accretion and outflow signatures in brown dwarfs}

In solar-type stars, the most straightforward way of identifying ongoing disk accretion (i.e., the CTT phase) is through the presence of optical and/or UV 'veiling' (filling in of photospheric absorption lines), which arises due to excess continuum emission from the hot accretion shock region. Unfortunately, extremely few BDs manifest measurable veiling; as we shall see in $\S 3$ and 4.1, this is due to their very small accretion rates.

Another widely used accretion diagnostic in solar-type stars is the equivalent width (EW) of the $H \alpha$ emission line, which is expected to arise both in the accretion funnel flow as well as in the accretion shock: $\mathrm{EW}[H \alpha]>10 \AA$ is usually adopted as signature of ongoing accretion in these stars, while smaller values signify only chromospheric emission. However, this cutoff cannot be extended to arbitrarily late spectral types such as young $\mathrm{BDs}$ (SpT $\gtrsim \mathrm{M} 6$ at ages of a few Myr). The underlying photospheric continuum at $H \alpha$ $(6563 \AA)$ lies in the Wien part of the spectrum in the M types, and thus declines drastically with decreasing temperature (later type). Thus even a small chromospheric $H \alpha$ flux can easily exceed $10 \AA$ in emission EW for very low-mass stars (VLMS) and BDs. To counter this problem, Martín (1998), Barrado y Navascués \& Martín (2003) and White \& Basri (2003) have proposed spectral-type dependent EW $[H \alpha]$ cutoffs for identifying accretion, based on the saturation EW of purely chromospheric $H \alpha$ emission observed as a function of spectral type.

White \& Basri (2003) also pointed out a much more straightforward way of identifying accretion, based on the profile of the $H \alpha$ emission. They noted that empirically, in highresolution spectra, the full-width of the $H \alpha$ line at $10 \%$ of the emission peak (三FW10) was always $\gtrsim 270 \mathrm{kms}^{-1}$ in accretors regardless of spectral type, from solar type stars down to the substellar limit (SpT $\sim \mathrm{M} 6-\mathrm{M} 7)$. In subsequent work, Jayawardhana et al. (2003) lowered this cutoff to FW10 $2200 \mathrm{kms}^{-1}$ for the BD regime (SpT > M6). The simple explanation for this behaviour is that the accretion flow arises from the disk edge at a few stellar radii, and thus attains nearly free-fall velocities in travelling to the central star/BD. Even without other line-broadening mechanisms (e.g., Stark), this implies fullwidths $\gtrsim 200-250 \mathrm{kms}^{-1}$ for the $\mathrm{H} \alpha$ line wings in low mass stars and BDs (Jayawardhana et al. 2003, Mohanty et al. 2005). One caveat here is that for the low accretion rates seen in BDs $(\S 3,4.1)$, Stark broadening of the line is not expected to be a strong factor, and 
the infall velocity appears to be the primary driver of the $H \alpha$ line-width (Muzerolle $e t$ al. 2000; Jayawardhana et al. 2003). Consequently, inclination effects can have a significant effect on the full-width: FW10 may fall below the $200 \mathrm{kms}^{-1}$ cutoff even in accreting BDs if the system is observed close to pole-on (Muzerolle et al. 2003; Mohanty et al. 2005). Nevertheless, combined with other line diagnostics such as permitted emission

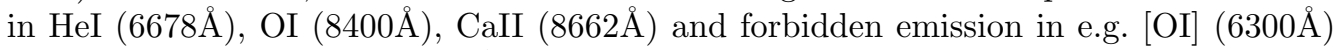
(see below), FW10 $200 \mathrm{kms}^{-1}$ provides a good conservative diagnostic for identifying accreting VLMS and BDs (Mohanty et al. 2005).

Various teams have now obtained high-resolution optical spectra of more than a hundred VLMS and BDs (SpT M5-M9.5: mass 0.1-0.015 $\mathrm{M}_{\odot}$ ) in nearby star-forming regions $(\mathrm{d} \lesssim 400 \mathrm{pc}$, age: $\lesssim 1-10 \mathrm{Myr}$ ), covering a major fraction of the known BDs in these regions (e.g., Muzerolle et al. 2003, 2005; Mohanty et al. 2003, 2005; Barrado y Navascués et al. 2004). Using the FW10 $[H \alpha]$ diagnostic above, scores of these sources have been clearly identified as accretors. Some of the salient qualitative features observed are (see Figs. 1, 2, 3):

(1) accretion is observed over the full range of $\mathrm{BD}$ masses, down to the lowest mass objects studied ( $\left.\sim 15 \mathrm{M}_{J u p}\right)$, approaching the planetary mass boundary;

(2) the broad line-wings of the $H \alpha$ accretion line-profiles indicate free-fall infall velocities, implying the same paradigm applicable to CTTs: magnetospherically channeled accretion flows from an inner disk edge at a few stellar radii;

(3) the $H \alpha$ line profiles in VLMS and BD accretors often evince asymmetries and timevariability analogous to those seen in higher-mass CTTs (see $\S 4.3$ );

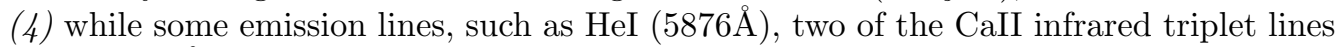
$(8498,8542 \AA)$ and of course $H \alpha$ itself, appear in both accreting as well as simply chromospherically active VLMS and BDs, others - HeI (6672 $)$, OI ( $8446 \AA)$ and one of the CaII infrared triplet lines $(8662 \AA)$ - appear to be present only in accreting VLMS and BDs (though not in all the latter), and are thus good secondary indicators of accretion when they are present, in addition to the $H \alpha$ FW10 diagnostic described above;

(5) forbidden emission lines such as [OI] (6300A), indicative of mass outflow in jets and winds, are observed in a few accreting BDs (see further below).

Accretion signatures are observed in VLMS and BDs in near-infrared spectra as well. In particular, accreting VLMS and BDs often exhibit $\mathrm{Pa} \beta$ and $\mathrm{Br} \gamma$ in emission, while merely chromospherically active VLMS and BDs do not (Fig. 2; Natta et al. 2004, 2006; Gatti et al. 2006). It is true that these lines are not as sensitive to accretion as $H \alpha$ : their smaller optical depth results in their absence in some low mass accretors that still exhibit broad $H \alpha$. Nevertheless, these near-infrared lines are especially important accretion diagnostics in regions of high extinction (e.g., $\rho$ Oph), where high-resolution optical spectroscopy is prohibitively time-consuming.

Finally, accretion signatures in CTTs are often accompanied by those of mass outflow in jets and winds. Indeed, from a theoretical perspective, accretion and outflow are inextricably linked, with the latter carrying away excess angular momentum from the infalling material. In the VLMS and BD regime, jet/wind indicators, such as emission in forbidden lines of [OI], [NII] and [SII] and blue-shifted absorption in $H \alpha$, have been identified in only a handful of sources (Figs. 1, 3; e.g., Barrado y Navascués et al. 2004; Whelan et al. 2005, 2007; Muzerolle et al. 2005; Mohanty et al. 2005). This is not too surprising: if the outflow rates are comparable to the infall ones (as theory suggests), then the very small accretion rates in the VLMS/BD regime would generally result in outflow signatures near/below current sensitivity limits. Indeed, in the one BD in which both accretion and outflow rates have been estimated from high-resolution spectra (LSRCrA-1: Barrado y Navascués et al. 2004; Mohanty et al. 2005), the outflow estimates 

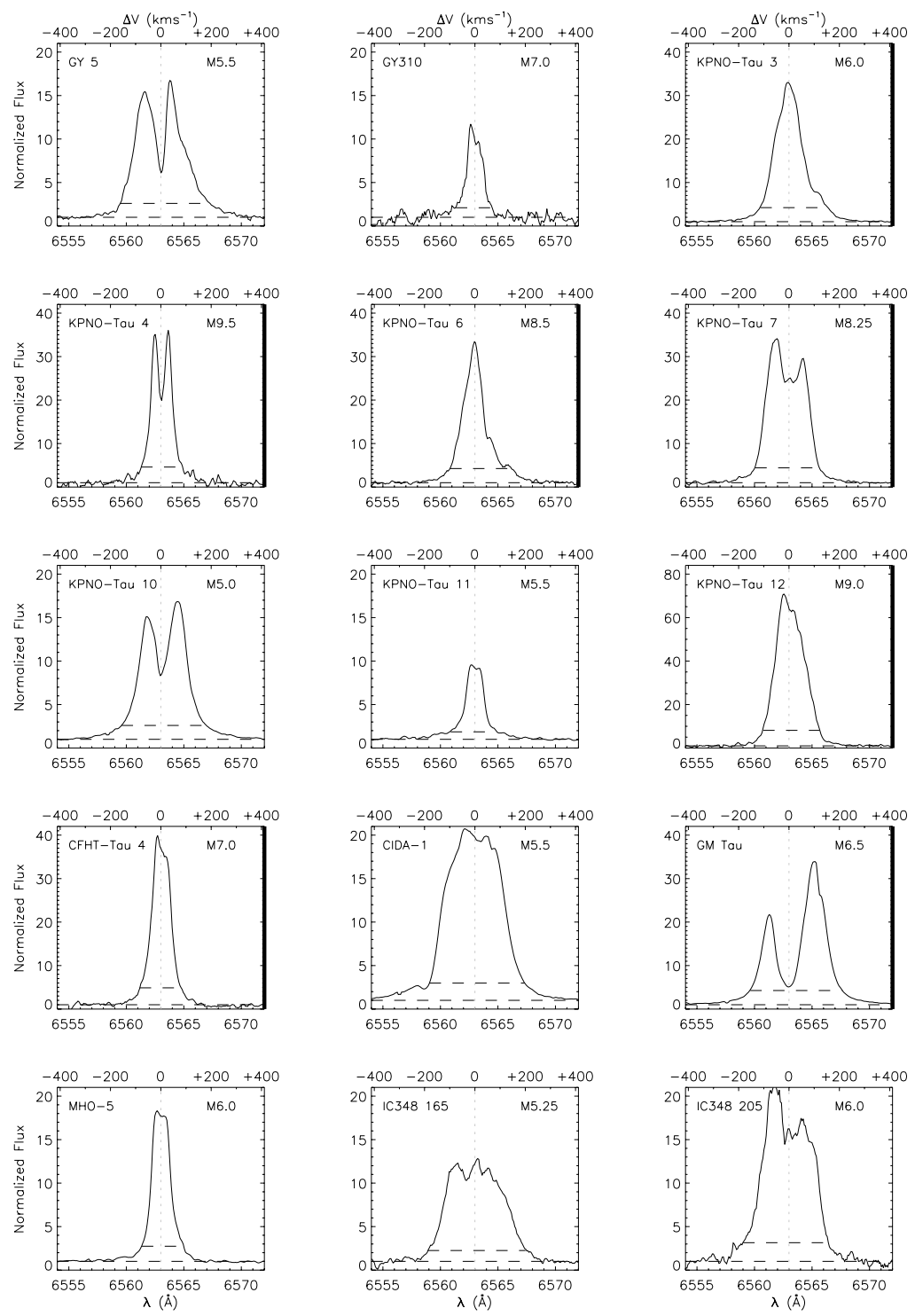

Figure 1. $H \alpha$ profiles in a sample of accreting BDs, exhibiting the broad line wings (except in a few pole-on cases), and line asymmetries due to geometrical and wind effects, associated with CTT-like magnetospheric accretion. From Mohanty et al. (2005).

range from an order of magnitude lower to comparable to the accretion rate, roughly consistent with theory. The jets in two BDs ( $\rho$ Oph 102 and 2MASS 1207-3932) have now also been spectro-astrometrically resolved (Whelan et al. 2005, 2007); the data suggest scaled-down versions of CTT jets, in agreement with the idea that the outflows scale with accretion. However, no BD jet has yet been spatially resolved in imaging.

\section{Measuring the accretion rates}

Accretion rates $(\dot{M})$ in CTTs are derived most straightforwardly from the accretion luminosity $\left(L_{a c c}\right)$, as measured from the excess continuum emission in the optical and 

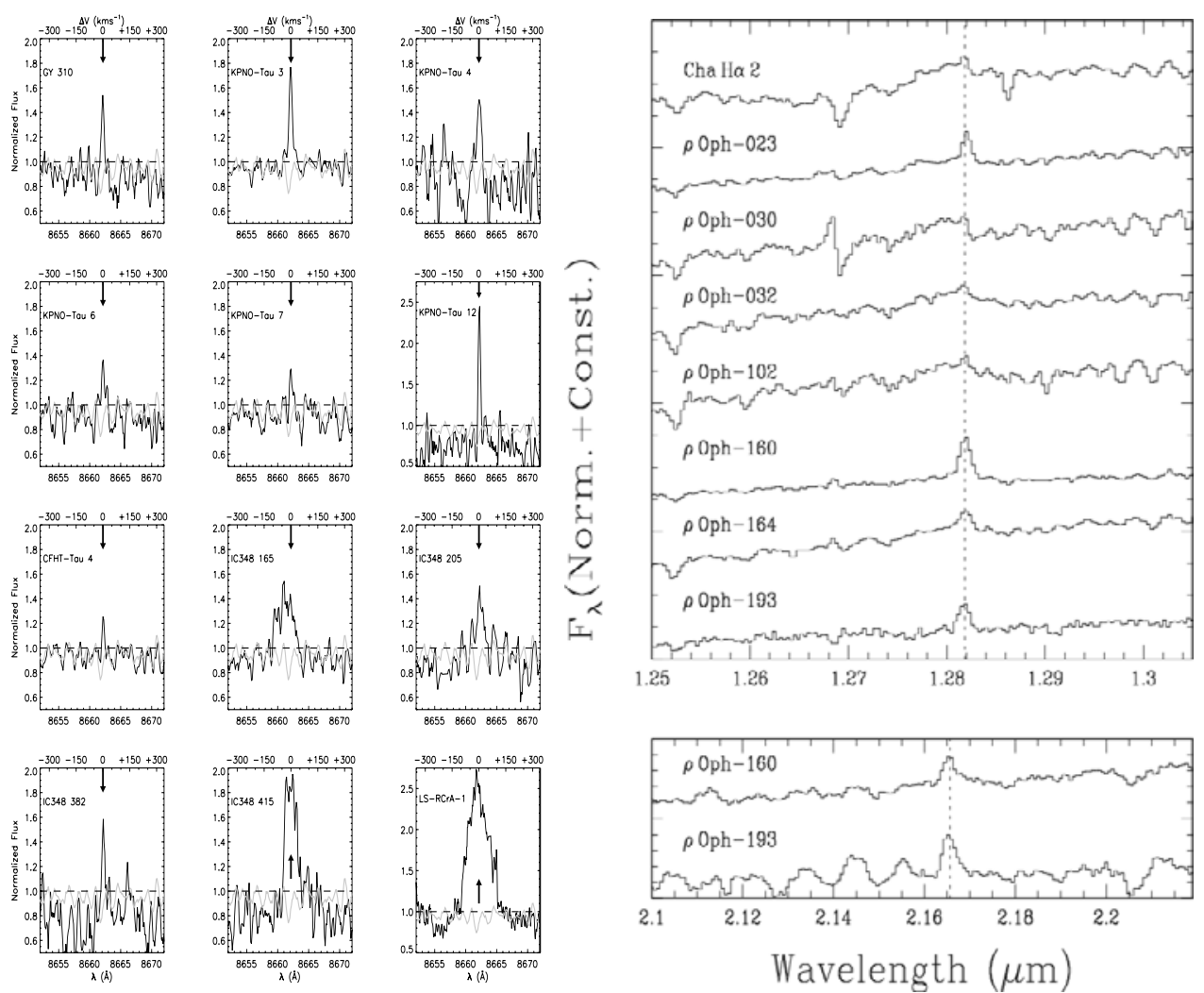

Figure 2. Left panels: CaII $8662 \AA$ emission (position marked with arrow) in a sample of BD accretors. Horizontal lines indicate the normalized continuum. The superimposed grey lines show the CaII region in non-accreting BDs of the same spectral type as the accretors, for comparison: the non-accretors do not evince any CaII 8662 emission. From Mohanty et al. (2005). Right panels: $\mathrm{Pa} \beta$ (top) and $\operatorname{Br} \gamma$ (bottom) emission detected in a sample of BDs. The dotted vertical lines show the emission position. From Natta et al. (2004).

UV. Unfortunately, as mentioned earlier, the infall rates in most BDs are too small to produce significant excess (Muzerolle et al. 2000 calculate that $\dot{M}<10^{-9} \mathrm{M}_{\odot} \mathrm{yr}^{-1}$ would be too small for any appreciable excess and veiling). Another technique is to model the $H \alpha$ line profile in detail (Muzerolle et al. 1998a). This is the method currently used most widely to directly measure $\dot{M}$ in VLMS and BDs (Muzerolle et al. 2000, 2003, 2005). In addition, two secondary methods have come into usage. The first relies on the empirically observed correlation between $\dot{M}$ and the emission flux in CaII infrared triplet lines. The correlation was first noted by Muzerolle et al. (1998b) for CTTs, with $\dot{M}$ measured from $L_{a c c}$; Mohanty et al. (2005) then showed that it extended into the BD regime as well (with $\dot{M}$ now measured from modeling the $H \alpha$ line), for the $8662 \AA$ line of the triplet. The other secondary technique relies on the analogous correlation observed between $\dot{M}$ and the flux in the $\mathrm{Pa} \beta$ and $\mathrm{Br} \gamma$ lines (Natta et al. 2004, 2006; Gatti et al. 2006). The advantage of these secondary methods, in the VLMS/BD regime, is that once the functional form of the correlation has been established, $\dot{M}$ can be derived from a simple measurement of line fluxes instead of a detailed and time-consuming modeling of the $H \alpha$ profile. The infrared $\mathrm{Pa} \beta$ and $\mathrm{Br} \gamma$ correlations moreover allow $\dot{M}$ to be measured for very extincted sources. 

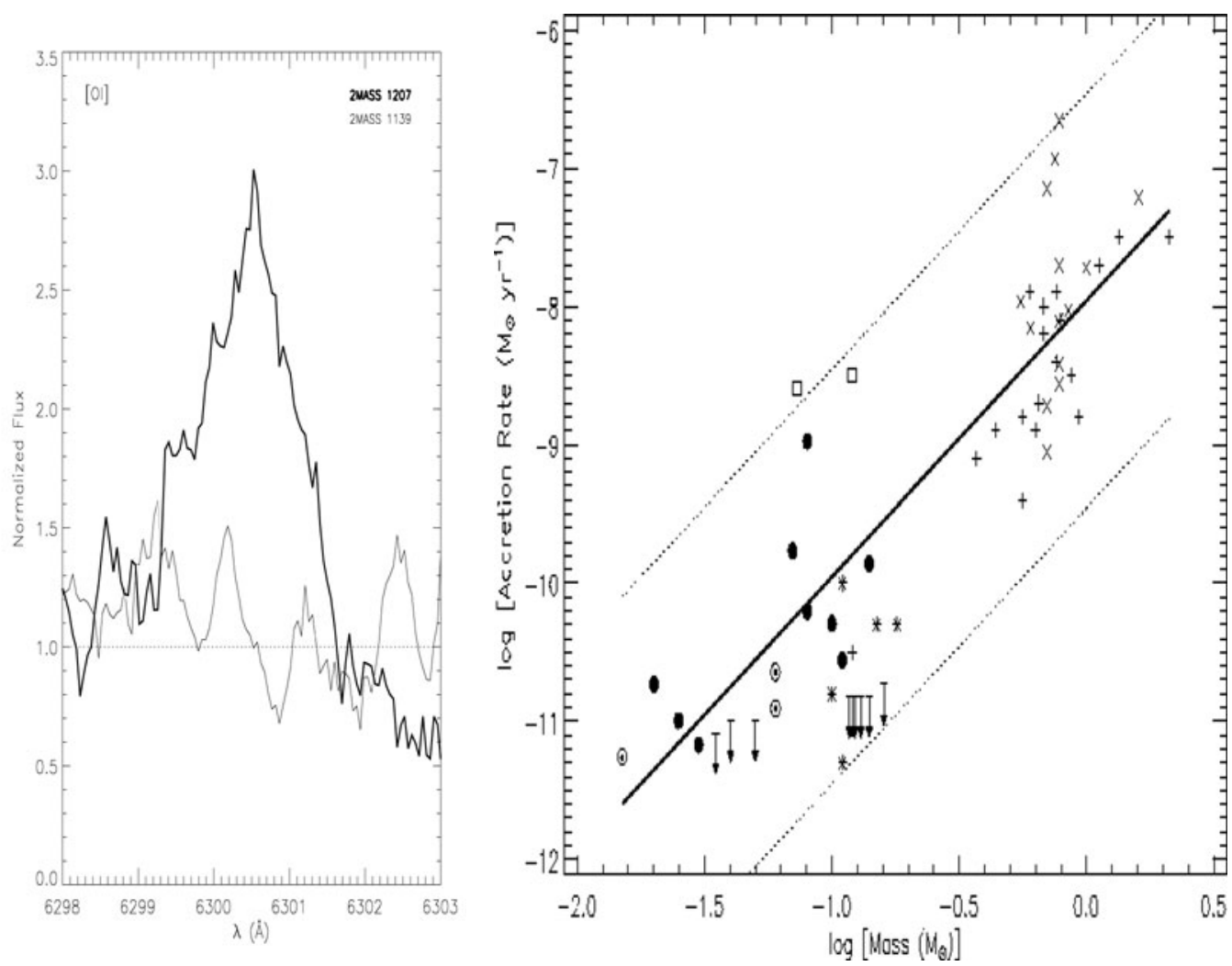

Figure 3. Left panel: Forbidden [OI] 6300A emission from the BD 2MASS1207-3932 (thick line), indicating the presence of an outflowing wind/jet. Thin line is the same spectral region from the non-accreting BD 2MASS1139, without any [OI], for comparison. Right panel: $\dot{M}$ vs $M_{*}$, from solar-mass CTTs down to $\sim 15 \mathrm{M}_{J u p}$ BDs. Symbols indicate the various studies in the compilation (see Mohanty et al. 2005 and references therein). Solid line is the formal fit to the data (excluding $\dot{M}$ upper limits), and follows almost exactly $\dot{M} \propto M_{*}^{2}$. Dashed lines are this fit vertically offset by $\pm 1.5 \mathrm{dex}$, to denote the upper/lower envelope of the trend. Both plots from Mohanty et al. (2005).

The drawbacks are threefold. First, from a theoretical standpoint, the basis of the correlations are not completely understood. In particular, the $\mathrm{CaII}, \mathrm{Pa} \beta$ and $\mathrm{Br} \gamma$ lines in CTTs appear to arise predominantly in the infalling funnel flow, while in BDs they seem to be produced only in the shock (Muzerolle et al. 1998b; Mohanty et al. 2005; Gatti et al. 2006). Thus, while the lines do probe accretion in both CTTs and BDs, it is not clear why the functional form of the relationship between $\dot{M}$ and the line fluxes should be similar in the two mass regimes. Nevertheless, this does not detract from the usefulness of these secondary techniques, since the correlations are empirically observed.

Second, the CaII, $\mathrm{Pa} \beta$ and $\mathrm{Br} \gamma$ lines are somewhat less sensitive to accretion than $H \alpha$ : they are absent for $\dot{M} \lesssim 10^{-11} \mathrm{M}_{\odot} \mathrm{yr}^{-1}$, while $H \alpha$ continues to be a viable diagnostic down to $\sim 10^{-12} \mathrm{M}_{\odot} \mathrm{yr}^{-1}$. Third, and most important, the viability of these secondary techniques is ultimately based on the accuracy of the $\dot{M}$ from $H \alpha$ modeling in the BD regime (which is how the correlations between $\dot{M}$ and line fluxes are established in the first place). While the modeling has become increasingly sophisticated, it has not been 
adequately tested against the most direct method - deriving $\dot{M}$ from $L_{a c c}$ - in BDs, due to the lack of significant continuum excess in the latter. The modeling also does not currently account for winds, which can affect the $H \alpha$ profile. Consequently, the $\dot{M}$ inferred at present for VLMS and BDs, either from $H \alpha$ modeling or the secondary techniques based on the latter, must be regarded as being somewhat imprecise, though probably correct to within a factor of $\sim 5$ (Muzerolle et al. 2003, 2005). In any case, this is the best that can be achieved at present. Using these methods, $\dot{M}$ have now been derived for scores of VLMS and BDs. The accretion rates are in the range $10^{-10^{-}}-10^{-12}$ $\mathrm{M}_{\odot} \mathrm{yr}^{-1}: 2-4$ orders of magnitude lower than the average in CTTs.

\section{Behaviour of the accretion rates}

\subsection{Dependence of $\dot{M}_{a c c}$ on central mass}

The accretion rates derived so far point to a clear correlation between $\dot{M}$ and the mass of the central object $\left(M_{*}\right)$, all the way from solar-mass stars down to the lowest mass BDs (Fig. 3). In particular, it appears that roughly, $\dot{M} \propto M_{*}^{2}$ (albeit with considerable scatter), over $\sim 4$ orders of magnitude in $\dot{M}$ and 2 orders in $M_{*}$ (Muzerolle et al. 2003, 2005; Natta et al. 2004, 2006; Mohanty et al. 2005).

The reasons for this relationship are unclear, though a few explanations have been offered. Padoan et al. (2005) argue that it results from Bondi-Hoyle accretion frrom the surrounding cloud onto the disk. In the latter picture, $\dot{M}_{B H} \propto M\left[\rho_{\infty} /\left(c_{\infty}^{2}+v_{\infty}^{2}\right)\right]$, where $\dot{M}_{B H}$ is the accretion rate onto the star+disk system from the surrounding medium, $M$ is the total mass of star+disk, and $\rho_{\infty}$ and $c_{\infty}$ are respectively the gas density and sound speed in the surrounding medium and $v_{\infty}$ the velocity of the medium relative to the star+disk system. This directly gives the correlation observed between infall rate and central mass, and explains the observed scatter in terms of the variability of stellar velocity and conditions in the cloud. The first drawback, however, is that Bondi-Hoyle accretion refers to infall from the cloud onto the star+disk, while the observed accretion rates are through the disk onto the star. It is not clear why the two should be related, except perhaps in a time-averaged sense. Moreover, there does not appear to be any systematic difference in $\dot{M}$, for stars of similar mass, between regions very with large differences in cloud properties and stellar velocities, whereas the Bondi-Hoyle scenario would predict a sytematic variation of a few orders of magnitude (e.g., Trumpler 37 versus Taurus: Sicilia-Aguilar et al. 2005; Hartmann et al. 2006).

Dullemond et al. (2006) instead suggest that the relationship arises due to the rotational properties of the original star-forming cores (specifically, they assume that all cores regardless of size are rotating at the same fraction of breakup velocity), combined with properties of viscous accretion disks. Their adopted conditions essentially result in two relationships $-M_{d i s k} \propto M_{*}^{2}$ and $\dot{M} \propto M_{d i s k}$ - which in combination produce the observed $\dot{M} \propto M_{*}^{2}$ correlation. However, the data appear substantially inconsistent with the $\dot{M}$ $\propto M_{\text {disk }}$ relation they expect. On the other hand, current disk masses are very insecure, due to large uncertainties in grain opacities; it is hence possible that this explanation for the $\dot{M}-M_{*}^{2}$ relation will appear more viable with improved disk masses.

Finally, Hartmann et al. (2006) suggest that the relationship arises due to a combination of factors that change with the mass regime: perhaps the lowest mass stars have the least massive disks, that can be thoroughly magnetically active with accretion driven throughout by the magneto-rotational instability, while higher mass stars have more massive disks which have magnetically inactive regions (dead zones) where gravitational instabilities may drive accretion. The viability of this hypothesis, like that of Dullemond 
et al. (2006) above, depends on obtaining more robust disk masses. It also predicts that disk evolution should proceed much more rapidly in the lowest mass sources, with $\dot{M}$ dropping off significantly faster with age in BDs than in higher-mass CTTs. The sample of VLMS and BD accretors as a function of age is too limited at this stage to test this claim (see $\$ 4.2$ ).

Finally, a proposed bias that is often brought up in this context deserves mention. Is it possible that the apparent $\dot{M}-M_{*}^{2}$ correlation results from an observational bias, wherein VLMS and BDs with very high accretion rates are not identified as such? Specifically, low mass sources with very large $\dot{M}$ would be heavily veiled and masquerade as higher mass stars, thereby escaping inclusion in the VLMS/BD sample. Including such sources would reduce the steepness of the accretion rate/mass relationship, and may remove the correlation altogether. However, this suggestion is probably not viable. A large fraction of young VLMS and BDs known today were originally identified in infrared surveys, and the effects of accretion-related veiling should be much smaller at such wavelengths than in the optical. Thus, sources with high veiling should be apparent by a significant mismatch between the optical and infrared spectral types. No such objects have been identified, implying that the $\dot{M}$ in VLMS and BDs is truly much lower than in CTTs, and that the observed $\dot{M}-M_{*}^{2}$ correlation does not result from a bias against low-mass sources with very large accretion rates. On the other hand, it is quite possible that sources with the lowest $\dot{M}$ in all mass regimes are not identified as accretors due to sensitivity limits, and the observed correlation thus represents the upper envelope of the true distribution.

\subsection{Temporal evolution of $\dot{M}_{a c c}$}

The number of VLMS and BDs observed in any given star-forming region still remains somewhat small. Nevertheless, some statistical conclusions have become apparent (Mohanty et al. 2005). First, in any given region, the fraction of VLMS and BDs that are accreting is comparable to that of CTTs. That is, there does not appear to be any sharp falloff in accreting objects in the VLMS/BD regime. Second, the fraction of VLMS/BDs with measurable accretion appears to decline with age, just as in solar-type stars, with much fewer accretors at $\gtrsim 5$ Myr than at $\sim 1$ Myr. Finally, accretion can continue in BDs over timescales comparable to that in CTTs: the oldest known BD accretor, the $\sim 25$ $\mathrm{M}_{J u p}$ source 2MASS 1207-3932, resides in the $\sim 10$ Myr-old TW Hydrae Association, which also harbors the oldest known CTTs (e.g., the eponymous star TW Hydra).

The evolution of the accretion rates in BDs is less well constrained. On the one hand, the $\dot{M}$ inferred for the oldest BD accretors, at 5-10 Myr, are among the lowest measured in the $\mathrm{BD}$ regime $\left(\sim 10^{-12} \mathrm{M}_{\odot} \mathrm{yr}^{-1}\right)$. On the other hand, equally small $\dot{M}$ have also been measured in much younger regions ( 1 Myr, e.g., Taurus), and the sample of $5-10 \mathrm{Myr}$ BD accretors is too small to make a meaningful comparison based on average $\dot{M}$ values. At younger ages $(<1-3 \mathrm{Myr})$, there does not appear to be a clear decline in average $\dot{M}$ with time; however, the sample at any given age is still somewhat too small to draw robust conclusions.

\subsection{Variability of line profiles and $\dot{M}_{a c c}$}

Accreting VLMS and BDs exhibit both photometric and spectroscopic (emission-line) variability, similar to that seen in CCTs, resulting from both stellar rotation and temporal changes in accretion rate/geometry (Scholz \& Eislöffel 2005; Mohanty et al. 2003; Scholz et al. 2005). In the most detailed study of variability in an accreting BD so far, Scholz et al. (2005) show that 2MASS 1207-3932 evinces quasi-periodic variability over timescales of hours to months, with a red-shifted absorption component in the $H \alpha$ line that is modulated by the rotation period of the star. The data suggest a change of about 
an order of magnitude in $\dot{M}$ over several weeks, and a close to edge-on system with an accretion funnel flow that rotates in and out of the line-of-sight. Extending such detailed studies to large samples of BDs in the future can clarify the physics and geometry of accretion flows in very low mass sources.

\section{Implications for brown dwarf formation}

To summarize, all the evidence above points to a very similar infancy for BDs and higher-mass stars. Disks and a disk accretion phase are equally ubiquitous in the stellar and substellar regimes, and the phenomenology and physics of the star-disk interaction magnetospherically channeled inflow from an inner disk edge at a few (sub)stellar radii, the presence of outflowing winds/jets along with inflow, a relationship between the accretion rate and central mass - are also the same in both regimes. Does this point to a similar formation process for BDs as for stars, in agreement with the 'turbulent fragmentation' scenario and in contrast to the 'ejection' hypothesis? While it is tempting to conclude so, the evidence for this is not yet ironclad.

In particular, the optical and infrared accretion and disk diagnostics arise within disk radii of $<1 \mathrm{AU}$. While the presence of disks and disk-accretion does rule out the most severe 'ejection' picture, wherein BD disks are completely sheared away, it does not rule out dynamical interactions which still allow some surrounding material to remain. In particular, current simulations imply that 'ejection' can still permit BD disks up to 10-20 AU in radius (Bate et al. 2003). Given the fator of $10^{2}-10^{4}$ smaller $\dot{M}$ in BDs compared to CTTs, this would also allow disk accretion in BDs to continue as long as in stars. What is required therefore is a measure of the true size of BD disks. Recent sub$\mathrm{mm} / \mathrm{mm}$ measurements do imply BD disk radii of at least $\sim 10 \mathrm{AU}$ (they may be much larger, but the data cannot determine whether this is so), which is nominally near the limit of the 'ejection' picture (Scholz et al. 2006). However, even if the disks were much smaller ( $\sim 1$ AU) initially, they would viscously expand to $\sim 10$ AU over Myr timescales. Only if BD disks are on average significantly larger than 10-20 AU can 'ejection' be ruled out confidently. Evidence for this does not yet exist. However, various investigations, some ongoing and others planned for the future, may help settle the question.

\section{Further formation constraints: recent and future observations}

One of the first tests proposed to test formation scenarios was to measure space velocities and spatial distribution: the initial 'ejection' model (Reipurth \& Clarke 2001) suggested that young BDs should have larger mean velocities than higher mass stars, and should therefore also be more spatially dispersed than the latter in star-forming regions. Various surveys (e.g. Luhman 2006) have shown that this is not the case: BDs have similar space velocities and distributions as stars. However, subsequent simulations showed that $\mathrm{BD}$ velocities would remain comparable to the average stellar velocity dispersion even for 'ejection' (Bate et al. 2003), so this is no longer a stringent test.

The existence of wide binaries is another possible test: the same dynamical interactions that would eject BDs and truncate their disks would also disrupt wide binaries. A few wide separation ( $\sim 40-250$ AU) BD binaries have now been discovered (e.g., Chauvin et al. 2004; Luhman 2004); at least for these systems, the 'ejection' scenario appears highly unlikely. The discovery of a large sample of such systems would argue against 'ejection' being the primary mode of BD formation. On the other hand, there is increasing evidence that the binary component separation decreases continuously as one moves to lower masses, and that BDs simply continue this trend (e.g., Kraus et al. 2006; Basri \& 
Reiners 2006). A lack of frequent wide binaries among BDs might therefore simply reflect binary formation mechanisms in general, without shedding light on the viability of the 'ejection' scenario for BDs in particular.

Measuring the true size of $\mathrm{BD}$ disks provides another means of discriminating between formation scenarios: as mentioned earlier, BD disks much larger than 10-20 AU would strongly argue against 'ejection'. Current modeling, based on fitting spectral energy distributions to observed sub-mm/mm dust emission, already suggests that the disks are at least as large as $~ 10$ AU. However, the number of BDs with such measurements is few (Scholz et al. 2006), and the modeling is also dependent on very uncertain dust opacities. Meoreover, as discussed, viscous spreading alone can produce $\sim 10$ AU disks even if they started out much smaller. A much better test would be to directly resolve the disks. The unprecedented sensitivity and spatial resolution of ALMA will be a great advantage in this area. However, even ALMA might be insufficient: while disks larger than $~ 10$ AU in the nearest star-forming regions $(\sim 150 \mathrm{pc})$ will nominally be resolved, material beyond $\sim 30 \mathrm{AU}$ in $\mathrm{BD}$ disks may be too cold to yield detectable sub-mm/mm emission, even with ALMA (Natta \& Testi 2006). Thus ALMA may not be able to decide whether BD disks are significantly larger than implied by ejection combined with viscous spreading.

Finally, the discovery of Class-0 BDs, i.e., proto-BDs embedded in their own isolated cores just like protostars, would provide strong support for the 'turbulent fragmentation' picture and argue against 'ejection'. Indeed, Spitzer has recently identified a number of very low luminosity objects (VELLOs), embedded within cores previously thought to be starless. Initial modeling suggests that these Class-0 objects have masses squarely in BD regime (e.g., Young et al. 2004; Huard et al. 2006; Bourke et al. 2006). These masses are rather insecure, however, since they depend on deconvolving the stellar, disk and envelope luminosity contributions; moreover, the accretion rates onto the central objects are poorly constrained. Studies are now underway to get more precise masses by obtaining infrared spectral types for the VELLOs, and also constrain the accretion rates by measuring the $\mathrm{Pa} \beta$ and $\mathrm{Br} \gamma$ emission fluxes. Naively, one expects that if the central masses are currently in the BD regime, and the measured accretion rates are also too low to allow them to eventually reach stellar masses over the normal lifetime of a core, then these must be true proto-BDs. However, reality may not be so simple. While the VELLO masses are themselves very low, the cores they are embedded in are of order a solar mass, i.e., not particularly small ones. It is difficult to imagine why a solar mass core would produce a BD-mass object, and not a low-mass star. Instead, it is possible that the accretion onto the central object is episodic, with long periods of low accretion punctuated by short bursts of intense accretion (as suggested by FU Orionis outbursts); most of the mass accumulated would be during the short bursts. In this case, a BD mass allied with a very low accretion rate would perhaps be the usual state of a low mass star during much of the Class-0 phase, with high accretion rates being statistically rare. Hence currently known VELLOs may not necessarily be proto-BDs, or be able to adjudicate between BD formation scenarios.

What the 'turbulent fragmentation' scenario really says is that BDs form out of gravitationally bound substellar-mass cores. Recent observations already indicate that cores with BD masses are indeed present in star-forming regions (e.g., Walsh et al. 2007; Greaves 2005). However, the bound nature of these cores is still uncertain; while the line-width data do suggest that some are gravitationally bound, the conclusion is not robust due to sensitivity issues. ALMA will provide a huge advance in this regard: it should be able to identify substellar-mass cores with ease, and even spatially resolve the nearest ones (Natta \& Testi 2006). The firm detection of isolated gravitationally bound BD-mass cores would be a clear indication that BDs can form just like stars, in agreement with 
the 'turbulent fragmentation' scenario. The further discovery of a proto-BD within one of these cores would of course be the final proof.

\section{Acknowledgements}

I acknowledge the support of the Spitzer Fellowship for this work.

\section{References}

Barrado y Navascués, D. \& Martín, E., 2003, AJ, 126, 2997

Barrado y Navascués, D., Mohanty, S. \& Jayawardhana, R., 2004, ApJ, 604, 284

Basri, G. \& Reiners, A., 2006, AJ, 132, 663

Bate, M. R., Bonnell, I. A., Bromm, V., 2003, MNRAS, 339, 577

Bourke, T. et al. , 2006, ApJ Letters, 649, L37

Chauvin, G. et al. , 2004, A\&A Letters, 425, L29

Dullemond, C., Natta, A., Testi, L., 2006, ApJ Letters, 645, L69

Gatti, T., Testi, L., Natta, A., Randich, S., \& Muzerolle, J., 2006, astro-ph/0609291

Greaves, J. S., 2005, Astronomische Nachrichten, 326, 1044

Hartmann, L., D'Alessio, P., Calvet, N., \& Muzerolle, J., 2006, ApJ, 648, 484

Huard,T. et al. , 2006, ApJ, 640, 391

Jayawardhana, R., Mohanty, S., \& Basri, G. 2003, ApJ, 592, 282

Kraus, A., White, R., \& Hillenbrand, L., 2006, ApJ, 649, 306

Luhman, K., 2004, ApJ, 614, 398

Luhman, K., 2006, ApJ, 645, 676

Martín, E. L., 1998, AJ, 115, 351

Mohanty, S., Jayawardhana, R., \& Barrado y Navascués, D., 2003, ApJ Letters, 593, L109

Mohanty, S., Jayawardhana, R., \& Basri, G., 2005, ApJ, 626, 498

Muzerolle, J., Calvet, N., \& Hartmann, L., 1998a, ApJ, 492, 743

Muzerolle, J., Hartmann, L., \& Calvet, N., 1998b, AJ, 116, 455

Muzerolle, J. et al. , 2000, ApJ Letters, 545, L141

Muzerolle, J., Hillenbrand, L., Calvet, N., Briceño,C., \& Hartmann, L., 2003, ApJ, 592, 266

Muzerolle, J., Luhman, K., Briceño, C., Hartmann, L., \& Calvet, N., 2005, ApJ, 625, 906

Natta, A., Testi, L., Muzerolle, J., Randich, S., Comerón, F., \& Persi, P., 2004, A\&A, 424, 603 [N04]

Natta, A., Testi, L., \& Randich, S., 2006, A\&A, 452, 245

Natta,A. \& Testi,L., 2006, Proceedings of "Science with ALMA: A new era for astrophyisics", http://www.oan.es/alma2006/contributions/Natta.pdf

Padoan, P. \& Nordlund,Å., 2004, ApJ, 617, 559

Padoan, P., Kritsuk, A., Norman, M., \& Nordlund,Å., 2005, ApJ Letters, 622, L61

Reipurth, B. \& Clarke, C., 2001, AJ, 122, 432.

Scholz, A. \& Eislöffel, J., 2005, A\&A, 429, 1007

Scholz, A., Jayawardhana, R., \& Brandeker, A., 2005, ApJ Letters, 629, L41

Scholz, A., Jayawardhana, R., \& Wood, K., 2006, ApJ, 645, 1498

Sicilia-Aguilar, A., Hartmann, L., Hernández, J., Briceño, C., \& Calvet, N., 2005, AJ, 130, 188

Walsh, A. et al. , 2007, ApJ, 655, 958

Whelan, E. et al. , 2005, Nature, 435, 652

Whelan, E. et al. , 2007, ApJ Letters, 659, L45

White, R. J. \& Basri, G., 2003, ApJ, 582, 1109

Young, C. et al. , 2004, ApJS, 154, 396 

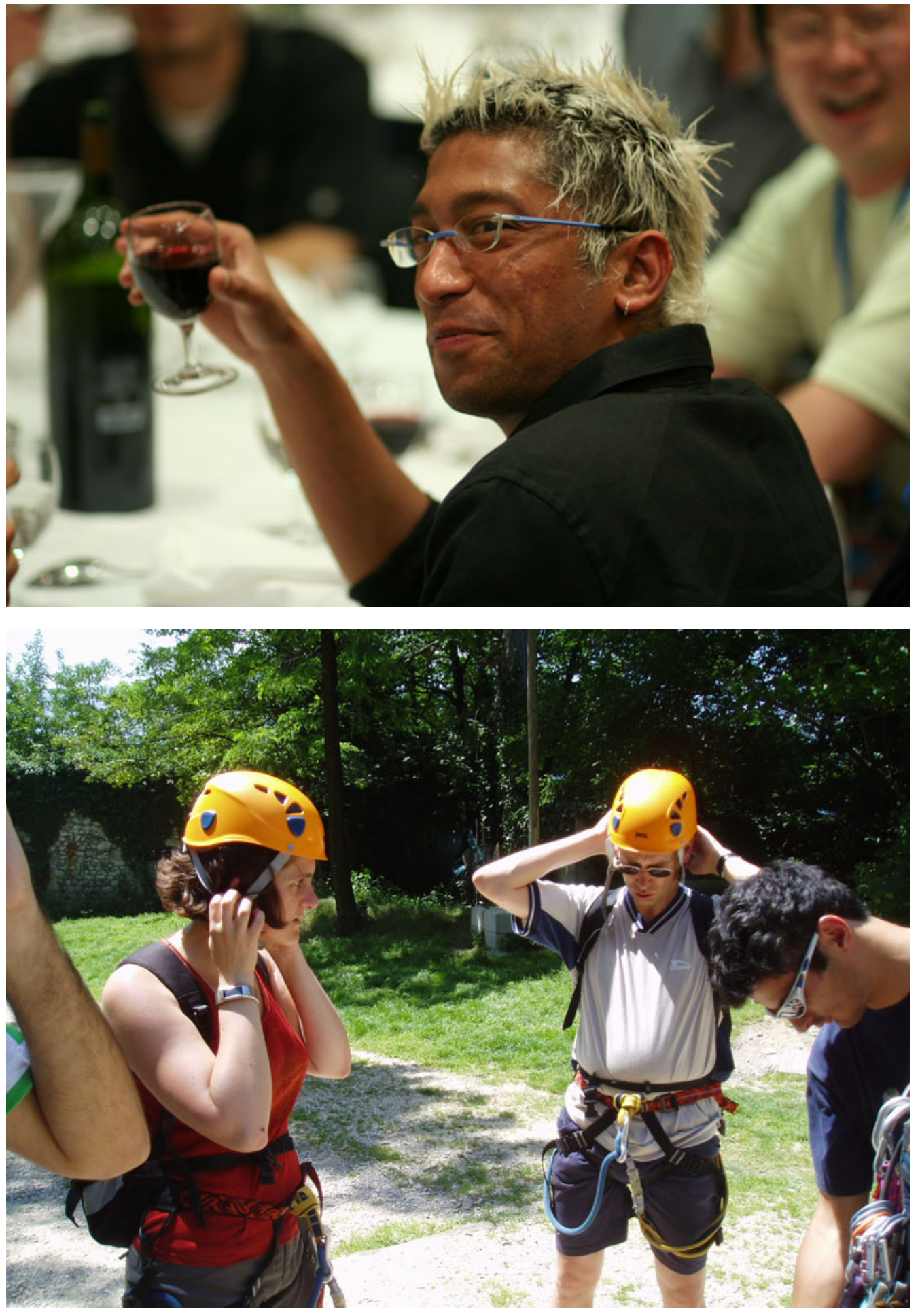